\title{
Detection and Classification of Muscle Activation in EMG Data Acquired by Myo Armband
}

\author{
Cengiz Tepe ${ }^{1}$, Mehmet Can Demir ${ }^{2 *}$ \\ ${ }^{1}$ Ondokuz Mayıs University, Faculty of Engineering, Electrical and Electronics Engineering, Samsun, Turkey (ORCID: 0000-0003-4065-5207) \\ ${ }^{2}$ Ondokuz Mayıs University, Faculty of Engineering, Electrical and Electronics Engineering, Samsun, Turkey (ORCID: 0000-0002-2372-4242)
}

(Bu yayın 26-27 Haziran 2020 tarihinde HORA-2020 kongresinde sözlü olarak sunulmuştur.)

(DOI: 10.31590/ejosat.779660)

ATIF/REFERENCE: Tepe, C. \& Demir, M. C. (2020). Detection and Classification of Muscle Activation in EMG Data Acquired by Myo Armband. Avrupa Bilim ve Teknoloji Dergisi, (Special Issue), 178-183.

\begin{abstract}
Electromyogram (EMG) signals are signals that contain information about contractions in the muscles. EMG signals are personal and express which muscles contract at what intensity. In detecting these signals, Myo armband has been used frequently in recent years. There are eight EMG sensors, accelerometer sensors and gyroscopes on the Myo armband. These eight EMG sensors settle on different muscles on the arm and measure the contraction intension of the muscles during gesture. In this way, the gesture using the information of which of the eight sensors is contracted can be recognized. Myo armband acquire EMG data with a sampling frequency of $200 \mathrm{~Hz}$. In this study, EMG data was acquired by repeating 10 times 4 different hand gestures by 4 subject by attaching Myo armband to the right forearm. First, a high pass filter was applied to eliminate the noise from the acquired data and then the times when the hand gesture started and ended were determined. The aim of this study is to propose a new method to the literature to find the start and the end times of hand gesture at this point. Five time domain features of the preprocessed EMG signals were extracted. These features were root mean squire (RMS), mean absolute value (MAV), zero crossing (ZC), waveform length (WL) and slope sign change (SSC). Sequential forward selection was made in order to find the most successful feature set among the extracted features. For classification, SVM and KNN algorithms were used. As a result of the study, SVM algorithm with the WL feature gave the best result and 98.75\% performance was achieved. The result obtained was compared with the studies in the literature. In addition, other methods in the literature used to find the times when the gesture starts and ends were applied to the dataset used in this study and the results were shown.
\end{abstract}

Keywords: Myo Armband, EMG Signals, feature extraction, feature selection, classification.

\section{Introduction}

Studies on wearable technologies have gained momentum recently. These studies focus on perceiving electrical activities in the human body. The signals caused by the contraction of the muscles are called Electromyogram (EMG). EMG are electrochemical activities that occur in the body as a result of muscle contraction. They occur as a result of electrical impulses being transmitted from the brain to the body via nerves. (Kocyigit \& Kilic, 2008; Uzunhisarcıklı, Çetinkaya, Fidan, \& Çalıkuşu, 2019).

When the studies conducted using the Myo armband in the literature are examined, many different applications are encountered. These are robot interface control (Morais, Neves, Masiero, \& de Castro, 2016), robotic arm control (Erin \& Boru, 2018), Myo integration and mobile application for the hearing impaired (Vachirapipop, Soymat, Tiraronnakul, \& Hnoohom, 2017), sign language recognition (Wibawa \& Sumpeno, 2017), sensor assisted EMG data recording system application (Kunapipat, Phukpattaranont, Neranon, \& Thongpull, 2018), classification of rock-paper-scissors gestures (Ploengpit \& Phienthrakul, 2016) and applications such as hand gestures classification (İşcan, Emeç, \& YeŞilldİrek, 2018).

When the studies in the literature using Myo armband are examined in terms of hand gestures; Examination of performance in hand amputations (Cognolato et al., 2018), real-time detection of hand gestures and muscle activation (Benalcázar et al., 2017), revealing the method based on the Support Vector Machine (SVM) for the recognition of hand gestures (Chen \& Zhang, 2019), real-time gestures

\footnotetext{
${ }^{*}$ Responsible Author Ondokuz May1s University, Faculty of Engineering, Electrical and Electronics Engineering, Samsun, Turkey, ORCID: 00000002-2372-4242, mehmetcan-demir@hotmail.com
} 
recognition based on dynamic segmentation and EMG (Yang, Pan, \& Li, 2017), Studies such as developing a new convolutional neural network (CNN) architecture developed by transfer learning techniques were encountered.(Cote-Allard et al., 2017).

The aim of this study is to propose a new method for the correct detection of the timess when the hand gesture starts and ends in an EMG data and to classify the EMG signals detected by this method. In the Section 2, the material and method related to how to determine the region of muscle activity and how the features are obtained are explained. Results and discussion obtained in the Section 3 are included. In the Section 4, the conclusion is summarized.

\section{Material ve Method}

\subsection{Data Acquisition}

Myo armband was used for data acquisition. Myo armband is one of the wearable technologies. It has eight EMG sensors, a gyroscope and accelerometer sensors. EMG data was acquired with Myo armband with $200 \mathrm{~Hz}$ sampling frequency and eight channels (Abduo \& Galster, 2015).

The dataset was created from four healthy subjects by repeating four gestures ten times. These four gestures were; Fist, spreadout wavein and waveout. The data was acquired by wearing the Myo armband on the right forearm while the subject was sitting upright in the chair. The data length was 5 seconds. In the 2nd second after the data acquisition started, the subject started to move hand and finished it voluntarily. The hand gesture took approximately 1 second.

In Figure 1, in order to see EMG signal differences between hand gestures, preprocessed EMG signals for each gesture are shown as 8 channels one after another.
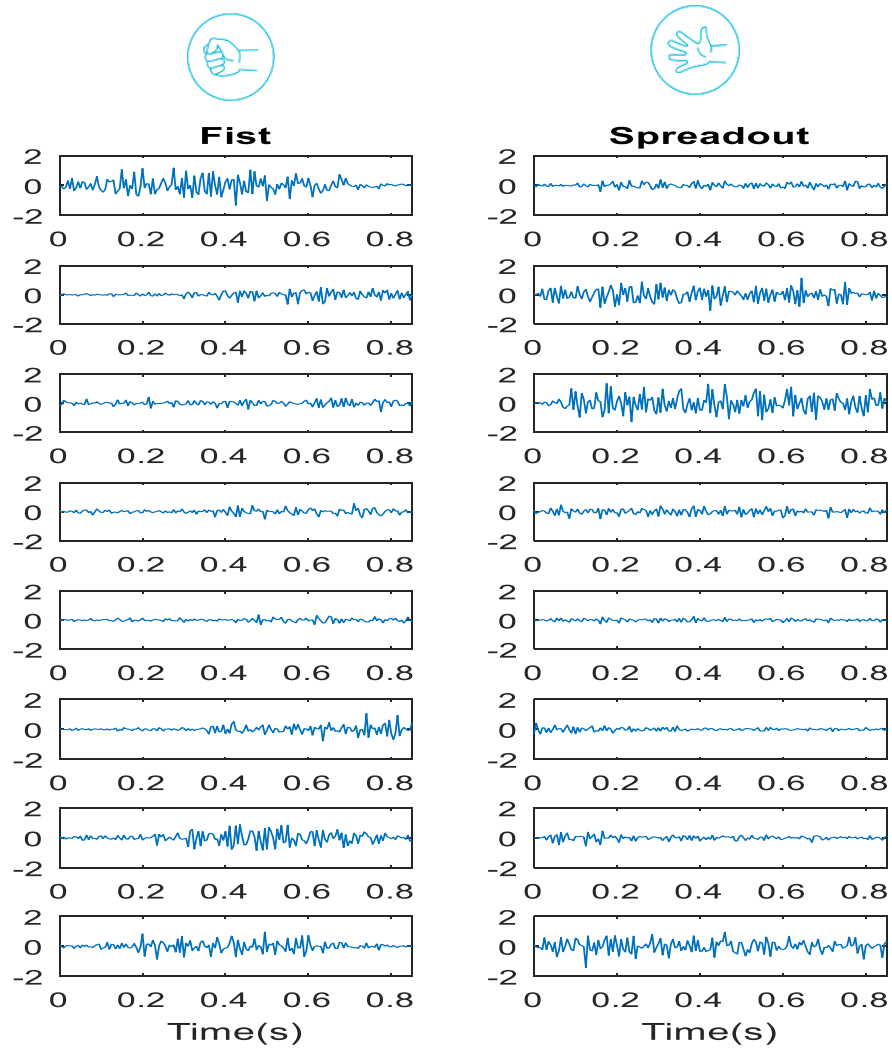
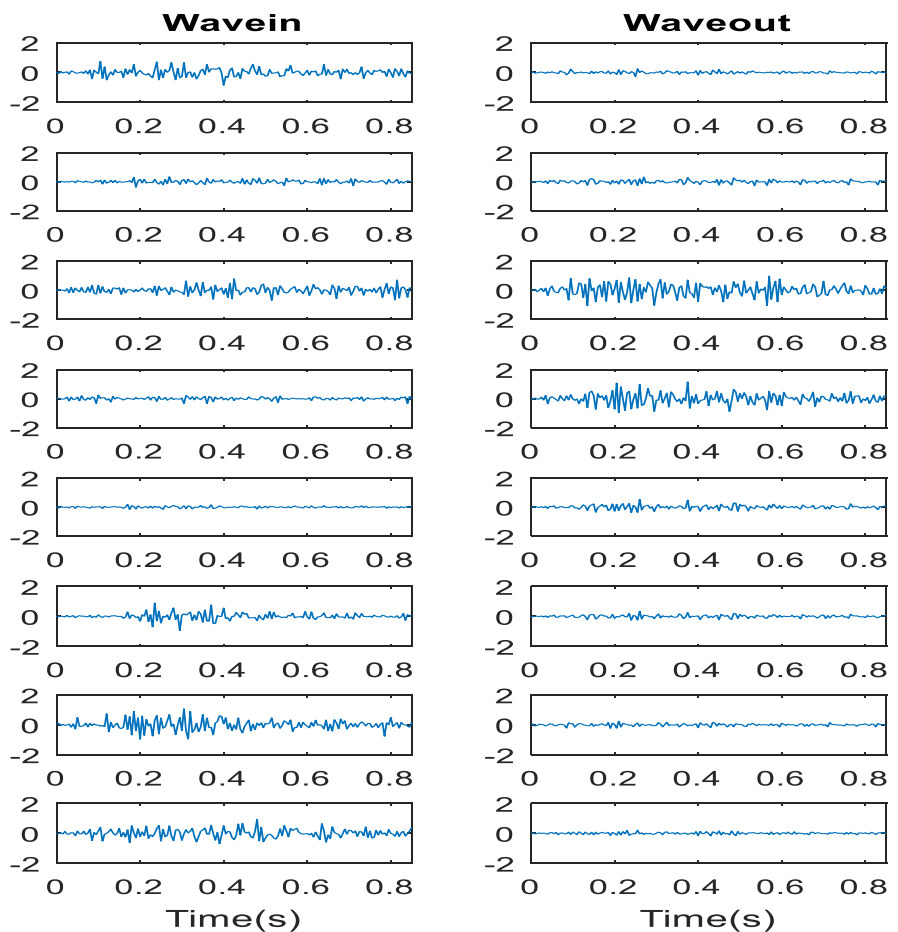

Figure 1. EMG Data For Four Gestures

\subsection{Finding the Start and the End Times of the Hand Gesture}

Before feature extraction of the EMG signal, the region in which the gesture occurs must be found and other regions must be eliminated from the signal. Thus, only the part expressing the gesture will be obtained. Before starting this process, the filter was applied to obtain the meaningful frequency range of the EMG signal. Different values are given in different studies for meaningful ranges of EMG signals (Barioul, Fakhfakh, Derbel, \& Kanoun, 2019; De Luca, Gilmore, Kuznetsov, \& Roy, 2010; Phinyomark \& Scheme, 2018). However, in all of these values, the upper limit is above $100 \mathrm{~Hz}$. Since the sampling frequency is $200 \mathrm{~Hz}$ in the EMG data acquired with Myo armband, the highest frequency of the EMG signals used in this study will be $100 \mathrm{~Hz}$ according to the Nyquist Theorem. For this reason, in the filtering process, a high pass filter with a cutoff frequency of $10 \mathrm{~Hz}$ was used in order to eliminate only low-frequency noise originating from the body. (Robertson, Caldwell, Hamill, Kamen, \& Whittlesey, 2013). 
The waveform length (WL) feature was used to determine the region where the gesture took place within the EMG signal. The signal is divided into $50 \%$ overlapping sliding window with $100 \mathrm{~ms}$ and Equation (1) expressing the waveform length is applied to all windows.

$$
\mathrm{WL}=\sum_{\mathrm{n}=1}^{\mathrm{N}-1}\left|\mathrm{x}_{\mathrm{n}+1}-\mathrm{x}_{\mathrm{n}}\right|
$$

$\mathrm{N}$ : number of samples in the window, $\mathrm{n}$ : calculated sample, $\mathrm{x}$ : amplitude

In order for the region of muscle activity to be detected automatically, it must first be determined where the signal starts. For this, the first 2 seconds without gesture in the signals were used to determine the threshold. First, the maximum value in the waveform length values in the first 2 seconds was determined. Then, by multiplying this value with $\sqrt{5}$, the threshold value was determined. After determining the threshold value, starting from the 2nd second, each value was checked and the first value that crosses the threshold value was accepted as the start of the gesture.

Based on the experience we have gained during these experiments, since contractions continue for a while after the gesture was completed by the nature of the human body, a second threshold was determined $\sqrt{ } 3$ times the first threshold to determine the end of the gesture. After determining the second threshold, the waveform length values were checked from the beginning of the gesture. The first value that falls below the threshold value was determined as the end. However, when the difference between the start and the end was very small, it was checked whether the next value after the detected value was within the threshold in order to prevent the detection of the wrong region due to the instantaneous drop and rise in the EMG signal. If not, this value was not accepted as a the start or the end and continued to be checked.

After determining the start and the end times, it should be found that the values representing the times of the start and the end correspond to the which samples in the EMG signal. For this, the information of the start and the end times were multiplied by the window step. Since $100 \mathrm{~ms}$ window length and $50 \%$ overlap was applied, the window step was $50 \mathrm{~ms}$, that was $0.05 \mathrm{~s}$. When we multiplied this value by the sampling frequency, there were 10 samples. In other words, by multiplying the start and end times information by 10 , it could be automatically detected on the EMG signal that the gesture starts at what value and ends at what value. Figure 2 shows the region of muscle activity in which gesture was detected on an EMG signal.

The values of $\sqrt{ } 5$ and $\sqrt{ } 3$ used in this method were found by trial and error method. The method was tested one by one on all EMG signals in the dataset, and the correct region was detected.

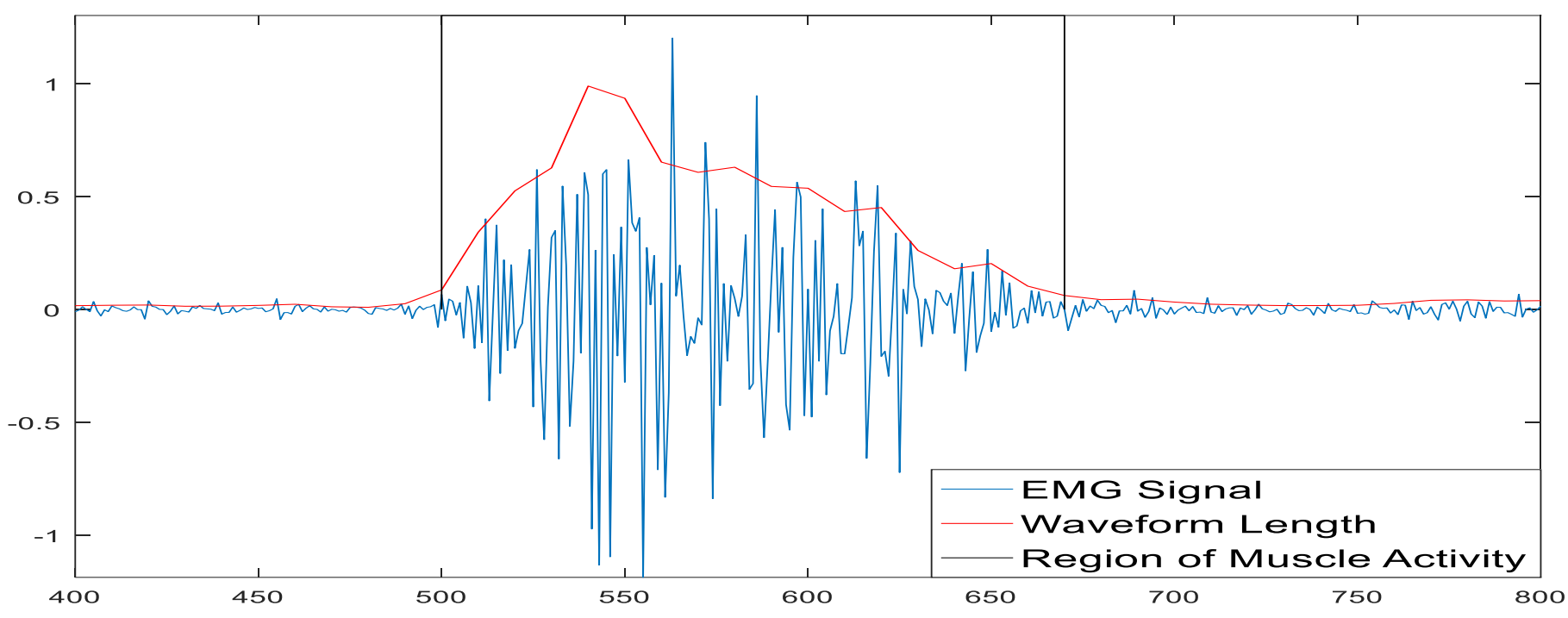

Figure 2. EMG Signal - Waveform Length - Region of Muscle Activity

\subsection{Feature Extraction and Feature Selection}

While extracting the features, the signal was divided into 50\% overlapping windows of $100 \mathrm{~ms}$ length and feature extraction was made for each window. Feature values found for a single signal were converted to a scalar value using the mean, one of the statistical parameters. Since data was acquired from eight channels in one gesture, the feature of a gesture consisted of a vector of $1 \times 8$. Therefore, for 4 gestures with 10 repetitions acquired from 4 subjects, a 160x8 feature vector was found.

When extracting the features, five time domain features were used. These were: root mean squire (RMS), mean absolute value (MAV), zero crossing (ZC), waveform length (WL) and slope sign change (SSC). The equations used when extracting features are shown in the equation (1-5) in Table 1(Phinyomark, Phukpattaranont, \& Limsakul, 2012). 
Table 1. Features

\begin{tabular}{|c|c|}
\hline $\mathrm{WL}=\sum_{\mathrm{n}=1}^{\mathrm{N}-1}\left|\mathrm{x}_{\mathrm{n}+1}-\mathrm{x}_{\mathrm{n}}\right|$ & $\mathrm{MAV}=\frac{1}{\mathrm{~N}} \sum_{\mathrm{n}=1}^{\mathrm{N}}\left|\mathrm{x}_{\mathrm{n}}\right|$ \\
\hline $\mathrm{ZC}=\sum_{\mathrm{n}=1}^{\mathrm{N}-1}\left[\operatorname{sign}\left(\mathrm{x}_{\mathrm{n}} * \mathrm{x}_{\mathrm{n}+1}\right) \cap\left(\mathrm{x}_{\mathrm{n}}-\mathrm{x}_{\mathrm{n}+1}\right)\right]$ \\
$\operatorname{sign}(\mathrm{x})= \begin{cases}1, & \mathrm{x} \geq 0 \\
0, & \mathrm{x}<0\end{cases}$ \\
\hline $\mathrm{RMS}=\sqrt{\frac{1}{\mathrm{~N}} \sum_{\mathrm{n}=1}^{\mathrm{N}} \mathrm{x}_{\mathrm{n}}^{2}}$
\end{tabular}

Among the 5 calculated features, sequential forward selection was made and the feature set giving the highest accuracy was selected (Stańczyk \& Jain, 2015). According to this method, the classification accuracy of each feature is checked one by one and it is determined that gives the highest accuracy in them. Then, others were added to the selected feature, and it was continued to look at the accuracy and this process was continued until the highest accuracy was reached.

\subsection{Classification}

In the classification phase, first of all, the feature matrix was randomly divided into two as training set and test set, containing an equal number of data. The separation was made so that the odd numbered rows go to the training set and the even numbered rows to the test set. Then, the label matrix was created by giving the label "1" for fist, "2" for spreadout, "3" for wavein and "4" for waveout, and added to the end of the training set.

The training set was used to train the model to be classified. The test set was used to test the training model. While calculating the training accuracies, k-fold cross-validation method was used and $\mathrm{k}=40$ was selected (Wong, 2015).

Support Vector Machine (SVM) and k-Nearest Neighbors (KNN) methods were used while classifying. SVM is based on the principle of determining the boundaries of the data of the two classes that are separated from each other in the most appropriate way. In $\mathrm{KNN}$, the closest $\mathrm{k}$ data to a test data is looked at, and the data belonging to which class is concentrated, the test data is assigned to that class.

\section{Results and Discussion}

In the feature selection, the classification accuracies of all the features were examined step by step and it was observed that the test accuracy did not exceed $98.75 \%$. The training and test accuracy of the first step of sequential forward selection are shown in Table 2 . Both training and test accuracy of the WL feature performed best with $98.75 \%$ when using the SVM classifier. For this reason, WL feature and SVM classifier were selected.

Table 2. Sequential Forward Selection First Step

\begin{tabular}{|c|c|c|c|}
\hline Feature & Training Accuracy & Test Accuracy & Classifier \\
\hline RMS & $97,5 \%$ & $98,75 \%$ & SVM $/ \mathrm{KNN}$ \\
\hline MAV & $97,5 \%$ & $98,75 \%$ & $\mathrm{SVM} / \mathrm{KNN}$ \\
\hline ZC & $46,25 \%$ & $52,5 \%$ & $\mathrm{SVM}$ \\
\hline WL & $98,75 \%$ & $98,75 \%$ & $43,75 \%$ \\
\hline SSC & $41,25 \%$ & SVM \\
\hline
\end{tabular}

The graphical distribution of the training set of the WL feature, which gives the highest performance, is shown in Figure 3. The channels that could show the best distribution while drawing the graphics were determined as 1-6-8 and the drawing was made on these channels. (İŞcan et al., 2018). Looking at the graph, it can be seen that the classes are separated from each other and gathered together. 


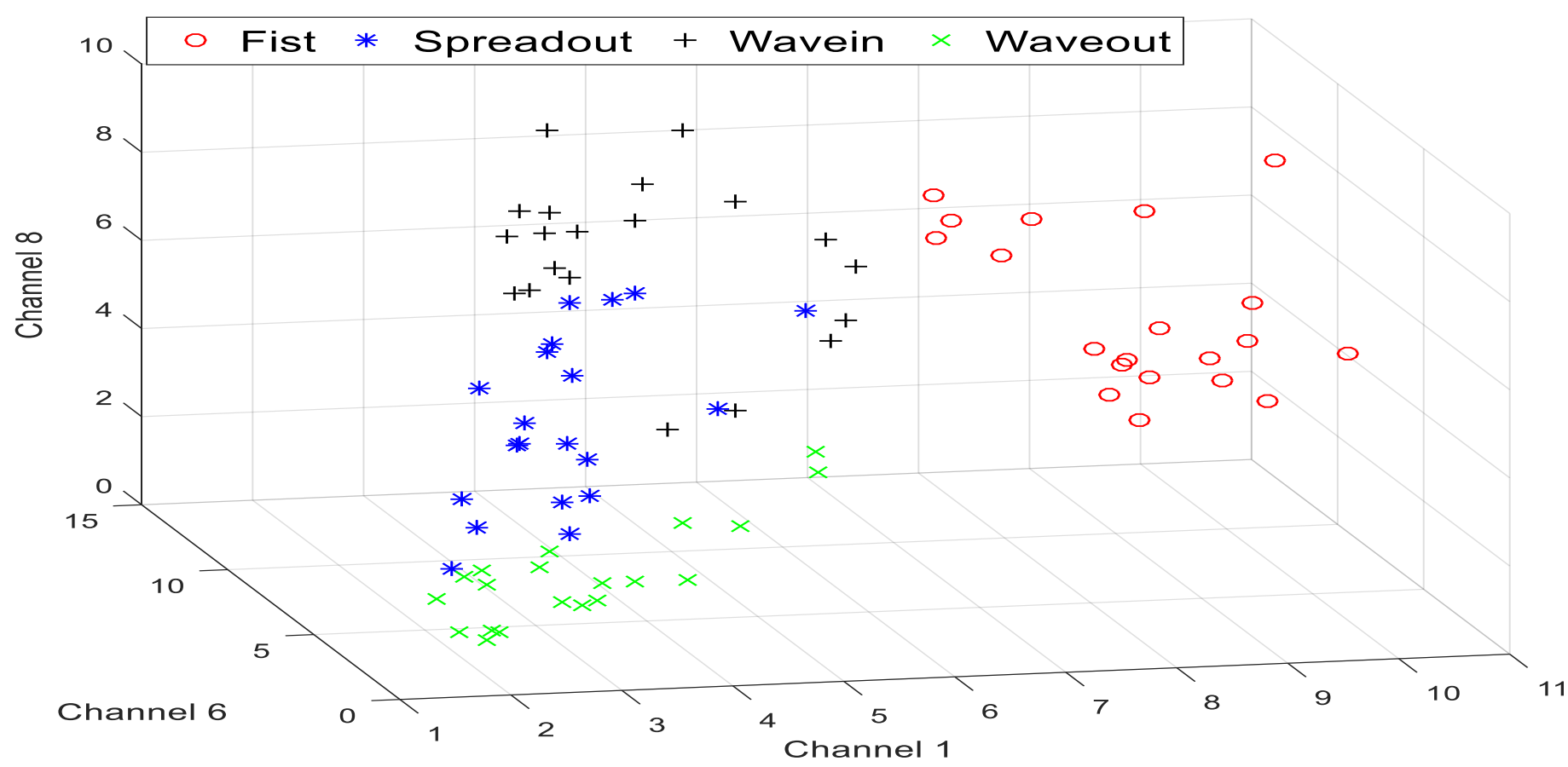

Figure 3. Graphical Distribution of WL Feature Training Set

Accuracy of eight studies with Myo armband is given in Table 3. In this study, a higher performance result is obtained from the seven studies in Table 3. There is a small difference of $0,25 \%$ between a study (Yang et al., 2017) in Table 3 and this study. This is probably because the dataset size they use is larger than in this study. Because there are 6 incorrect classifications in the confusion matrix. In this study, there is one incorrect classification.

Table 3. Performance Comparison With Other Studies With Myo Armband In The Literature

\begin{tabular}{|c|c|c|c|}
\hline Study & Classified Gestures & Classifier & Highest Performance \\
\hline (Wibawa \& Sumpeno, 2017) & 10 different sign language gestures & Naive Bayes & $98,63 \%$ \\
\hline (Kunapipat et al., 2018) & $\begin{array}{c}3 \text { different hand gestures and } 10 \\
\text { different arm movements }\end{array}$ & SVM & $91,28 \%$ \\
\hline (IŞcan et al., 2018) & 4 different hand gestures & Dynamically Structural Neural & Networks \\
\hline (Cognolato et al., 2018) & 5 different hand gestures & Myo Armband Classifier & $97,2 \%$ \\
\hline (Benalcázar et al., 2017) & 5 different hand gestures & KNN & $89,5 \%$ \\
\hline (Chen \& Zhang, 2019) & 5 different hand gestures & SVM & $89 \%$ \\
\hline (Yang et al., 2017) & 6 different hand gestures & GMM-HMM & $99 \%$ \\
\hline (Cote-Allard et al., 2017) & 7 different hand gestures & Convolutional Neural Networks & (CNN) \\
\hline This Study & 4 different hand gestures & SVM & $97,81 \%$ \\
\hline
\end{tabular}

In Table 4, other methods of finding the region of muscle activity in EMG signals (Wahid, Tafreshi, Al-Sowaidi, \& Langari, 2018; Yang et al., 2017) were applied to the dataset in this study and the results are shown. The method proposed in this study gave higher performance than these two methods.

Table 4. In other studies in the literature, the application of the methods of finding the start and the end times of the EMG signal to the dataset

\begin{tabular}{|c|c|c|}
\hline Study & Training Accuracy & Test Accuracy \\
\hline (Yang et al., 2017) & $28,75 \%$ & $35 \%$ \\
\hline (Wahid et al., 2018) & $90 \%$ & $87,5 \%$ \\
\hline
\end{tabular}




\section{Conclusion}

In this study, a method proposal has been put forward for determining the region of muscle activity in EMG signals. The EMG signal determined by this method was classified by extracting the features and $98.75 \%$ accuracy was obtained. It is aimed to expand the dataset and send the test data in real time in the future studies.

\section{References}

Abduo, M., \& Galster, M. (2015). Myo gesture control armband for medical applications.

Barioul, R., Fakhfakh, S., Derbel, H., \& Kanoun, O. (2019). Evaluation of EMG Signal Time Domain Features for Hand Gesture Distinction. Paper presented at the 2019 16th International Multi-Conference on Systems, Signals \& Devices (SSD).

Benalcázar, M. E., Motoche, C., Zea, J. A., Jaramillo, A. G., Anchundia, C. E., Zambrano, P., . . . Pérez, M. (2017). Real-time hand gesture recognition using the myo armband and muscle activity detection. Paper presented at the 2017 IEEE Second Ecuador Technical Chapters Meeting (ETCM).

Chen, W., \& Zhang, Z. (2019). Hand Gesture Recognition using sEMG Signals Based on Support Vector Machine. Paper presented at the 2019 IEEE 8th Joint International Information Technology and Artificial Intelligence Conference (ITAIC).

Cognolato, M., Atzori, M., Faccio, D., Tiengo, C., Bassette, F., Gassert, R., \& Muller, H. (2018). Hand Gesture Classification in Transradial Amputees Using the Myo Armband Classifier* This work was partially supported by the Swiss National Science Foundation Sinergia project\# 410160837 MeganePro. Paper presented at the 2018 7th IEEE International Conference on Biomedical Robotics and Biomechatronics (Biorob).

Cote-Allard, U., Fall, C. L., Campeau-Lecours, A., Gosselin, C., Laviolette, F., \& Gosselin, B. (2017). Transfer learning for sEMG hand gestures recognition using convolutional neural networks. Paper presented at the 2017 IEEE International Conference on Systems, Man, and Cybernetics (SMC).

De Luca, C. J., Gilmore, L. D., Kuznetsov, M., \& Roy, S. H. (2010). Filtering the surface EMG signal: Movement artifact and baseline noise contamination. Journal of biomechanics, 43(8), 1573-1579.

Erin, K., \& Boru, B. (2018). EMG ve jiroskop verileri ile endüstriyel robot kolunun gerçek zamanlı kontrolü. Sakarya University Journal of Science, 22(2), 509-515.

İŞcan, M., Emeç, C., \& YeŞİldİrek, A. (2018). Hand gesture movement classification based on dynamically structured neural network. Paper presented at the 2018 Electric Electronics, Computer Science, Biomedical Engineerings' Meeting (EBBT).

Kocyigit, Y., \& Kilic, I. (2008). Using LBG algorithm for extracting the features of EMG signals. Paper presented at the 2008 IEEE 16th Signal Processing, Communication and Applications Conference.

Kunapipat, M., Phukpattaranont, P., Neranon, P., \& Thongpull, K. (2018). Sensor-assisted EMG data recording system. Paper presented at the 2018 15th International Conference on Electrical Engineering/Electronics, Computer, Telecommunications and Information Technology (ECTI-CON).

Morais, G. D., Neves, L. C., Masiero, A. A., \& de Castro, M. C. F. (2016). Application of Myo Armband System to Control a Robot Interface. Paper presented at the BIOSIGNALS.

Phinyomark, A., Phukpattaranont, P., \& Limsakul, C. (2012). Feature reduction and selection for EMG signal classification. Expert systems with applications, 39(8), 7420-7431.

Phinyomark, A., \& Scheme, E. (2018). A feature extraction issue for myoelectric control based on wearable EMG sensors. Paper presented at the 2018 IEEE Sensors Applications Symposium (SAS).

Ploengpit, Y., \& Phienthrakul, T. (2016). Rock-paper-scissors with Myo Armband pose detection. Paper presented at the 2016 International Computer Science and Engineering Conference (ICSEC).

Robertson, G. E., Caldwell, G. E., Hamill, J., Kamen, G., \& Whittlesey, S. (2013). Research methods in biomechanics: Human kinetics.

Stańczyk, U., \& Jain, L. C. (2015). Feature selection for data and pattern recognition: Springer.

Uzunhisarcıklı, E., Çetinkaya, M. B., Fidan, U., \& Çalıkuşu, İ. (2019). Investigation of EMG Signals in Lower Extremity Muscle Groups During Robotic Gait Exercises. Avrupa Bilim ve Teknoloji Dergisi, 109-118.

Vachirapipop, M., Soymat, S., Tiraronnakul, W., \& Hnoohom, N. (2017). An integration of Myo Armbands and an android-based mobile application for communication with hearing-impaired persons. Paper presented at the 2017 13th International Conference on Signal-Image Technology \& Internet-Based Systems (SITIS).

Wahid, M. F., Tafreshi, R., Al-Sowaidi, M., \& Langari, R. (2018). Subject-independent hand gesture recognition using normalization and machine learning algorithms. Journal of computational science, 27, 69-76.

Wibawa, A. D., \& Sumpeno, S. (2017). Gesture Recognition for Indonesian Sign Language Systems (ISLS) Using Multimodal Sensor Leap Motion and Myo Armband Controllers Based-on Naïve Bayes Classifier. Paper presented at the 2017 International Conference on Soft Computing, Intelligent System and Information Technology (ICSIIT).

Wong, T.-T. (2015). Performance evaluation of classification algorithms by k-fold and leave-one-out cross validation. Pattern Recognition, 48(9), 2839-2846.

Yang, J., Pan, J., \& Li, J. (2017). sEMG-based continuous hand gesture recognition using GMM-HMM and threshold model. Paper presented at the 2017 IEEE International Conference on Robotics and Biomimetics (ROBIO). 